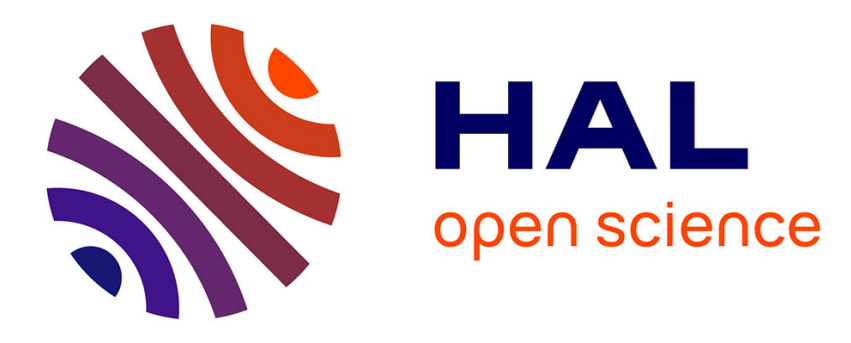

\title{
Finite volume schemes for two phase flow in porous media
}

\author{
Robert Eymard, Thierry Gallouët
}

\section{To cite this version:}

Robert Eymard, Thierry Gallouët. Finite volume schemes for two phase flow in porous media. Computing and Visualization in Science, 2004, 7, pp.31-40. hal-00003433

\section{HAL Id: hal-00003433 \\ https://hal.science/hal-00003433}

Submitted on 1 Dec 2004

HAL is a multi-disciplinary open access archive for the deposit and dissemination of scientific research documents, whether they are published or not. The documents may come from teaching and research institutions in France or abroad, or from public or private research centers.
L'archive ouverte pluridisciplinaire HAL, est destinée au dépôt et à la diffusion de documents scientifiques de niveau recherche, publiés ou non, émanant des établissements d'enseignement et de recherche français ou étrangers, des laboratoires publics ou privés. 


\title{
FINITE VOLUME SCHEMES FOR TWO-PHASE FLOW IN POROUS MEDIA
}

\author{
ROBERT EYMARD* AND THIERRY GALLOUËT ${ }^{\dagger}$
}

\begin{abstract}
The system of equations obtained from the conservation of multiphasic fluids in porous media is usually approximated by finite volume schemes in the oil reservoir simulation setting. The convergence properties of these schemes are only known in a few simplified cases. The aim of this paper is to present some new results of convergence in more complex cases. These results are based on an adaptation of the $\mathrm{H}$-convergence notion to the limit of discrete approximates.
\end{abstract}

Key words. finite volume schemes, two-phase flow, porous media, H-convergence.

AMS subject classifications. 35K65, 35K55.

1. Introduction. In the oil recovery engineering, numerical simulation is used to predict the displacements and transformations of the fluids within the reservoir. Since an accurate description of the thermodynamical properties of the fluids is essential, the engineers commonly use a finite volume scheme, in addition with a finite difference scheme for the computation of the fluxes between two neighboring cells. But the mathematical properties of such a scheme are as complex as the scheme is close to the engineering features and the convergence of the scheme, as the size of the discretization tends to zero, has only recently been studied. The aim of this paper is to give some new results in this direction. Since a complete oil reservoir model is too complex, we restrict our study to the following case: oil and water are assumed to be incompressible immiscible fluid phases with a common pressure, and the reservoir is supposed to be a homogeneous isotropic domain. Following [3], we express the conservation of the two phases in a domain $\Omega \subset \mathbb{R}^{N}(N=2$ or 3$)$ which represents the porous medium in this particular case, using Darcy's law, by the following equations:

$$
\begin{array}{ll}
\frac{\partial[\Phi(u) s]}{\partial t}-\operatorname{div}[\gamma(s) \lambda(s) \nabla u] & =S_{1}(u, s), \\
\frac{\partial[\Phi(u)(1-s)]}{\partial t}-\operatorname{div}[(1-\gamma(s)) \lambda(s) \nabla u] & =S_{2}(u, s),
\end{array}
$$

with some initial and boundary conditions. In (1.1), $u$ represents the common pressure of the two phases (if $N=3$ or if the physical domain is included in a tilted plane, this pressure is then an approximation of a common hydraulic head), the function $\Phi(u)$ (the porosity of the medium) is a strictly positive non decreasing function, used to approximate the coupling effects between the flows in the porous medium and the mechanical strain of the porous skeleton (it would be more rigorous to simultaneously solve the conservation equations and the mechanical equilibrium equations, but such a procedure, which is highly expensive from a computational point of view, is only done in an exceptional geological framework). The notation $s$ represents the saturation of the water phase, $\gamma(s)$ is a nondecreasing function which is called the "fractional flow", with $\gamma(0)=0$ and $\gamma(1)=1$, the positive function $\lambda(s)$ is the "total mobility" of the two phases (the sum of the mobility of water and the mobility of oil), the functions $S_{1}$ and $S_{2}$ represent some volumic source terms which approximate the flow rate due to the wells. The existence of a solution to (1.1) is an open problem if the function $\lambda$ is not reduced to a constant and must be discussed if $S_{1}(u, s)+S_{2}(u, s)$ depends on $s$. The convergence of the industrial scheme has recently been studied in the only case of a constant function $\lambda$ (see for example [7], [21] [15]). The aim of this paper is to study

\footnotetext{
*Université de Marne-la-Vallée, F-77454 Marne-la-Vallée Cedex, France(eymard@univ-mlv.fr).

${ }^{\dagger}$ CMI, Université de Provence F-13453 Marseille, France (gallouet@cmi.univ-mrs.fr).
} 
the convergence properties of finite volume schemes in the case where the function $\lambda$ is not a constant function. This problem appears to be very close to the study of the convergence, as $\varepsilon \longrightarrow 0$, of the solution of the problem

$$
\begin{array}{ll}
\frac{\partial\left[\Phi\left(u_{\varepsilon}\right) s_{\varepsilon}\right]}{\partial t}-\operatorname{div}\left[\gamma\left(s_{\varepsilon}\right) \lambda\left(s_{\varepsilon}\right) \nabla u_{\varepsilon}\right]-\varepsilon \Delta s_{\varepsilon} & =S_{1}\left(u_{\varepsilon}, s_{\varepsilon}\right) \\
\frac{\partial\left[\Phi\left(u_{\varepsilon}\right)\left(1-s_{\varepsilon}\right)\right]}{\partial t}-\operatorname{div}\left[\left(1-\gamma\left(s_{\varepsilon}\right)\right) \lambda\left(s_{\varepsilon}\right) \nabla u_{\varepsilon}\right]+\varepsilon \Delta s_{\varepsilon} & =S_{1}\left(u_{\varepsilon}, s_{\varepsilon}\right)
\end{array}
$$

where the additional term $\varepsilon \Delta s_{\varepsilon}$ stands for a diffusive term, which is similar to the diffusion added by the upstream weighted numerical schemes. Such a diffusive term is slightly different from that which comes from the introduction of a capillary pressure term, producing some degeneration similar to that of the porous media equation (see [1], [2], [11] and [5] for the existence of a solution of the continuous problem and see [10] for the proof of the convergence of a finite volume scheme).

An incomplete study of the convergence of the industrial finite volume scheme, used for the approximation of (1.1), is presented in this paper for both the steady and the transient cases. We state here that the approximate pressure strongly converges to the solution of a parabolic problem whose coefficients are obtained by the same method as the classical H-convergence proof (following [17], [14], [19] or [18]) whereas the approximate saturation only converges in a weak sense (namely in the weak $\star$ topology of $L^{\infty}$ ). The use, in the discrete setting, of a notion similar to H-convergence is natural: indeed, the existence of a limit as $\varepsilon \longrightarrow 0$ to the family of pressures $\left(u_{\varepsilon}\right)_{\varepsilon>0}$, solution to the sum of the equations (1.2), immediately results from the H-convergence background. Note that an extension of this background to a discrete setting has already been performed, see [16] and mainly [12] for the proof of the existence of a "H-limit" to a subsequence of a sequence of discrete elliptic operators, using regular structured grids and finite differences. The objective is here to study the limit of a sequence of finite volume approximations on general meshes, whereas the discrete diffusion results from the coupling of the two discrete conservation equations. The fact that the two unknowns are computed in the same grids makes different, in the general case, the notion of continuous and discrete $\mathrm{H}$-limits, which suggests to distinguish the vocabulary devoted to both notions.

It is also interesting to notice that the question of the independence of these limit coefficients on the way that some diffusion is added in (1.1) is not known. It is however clear that the limit $(u, s)$ of the numerical scheme or of the parabolic regularization (namely (1.2)) is a solution of (1.1) if a strong convergence result can be proved for the saturation. This sufficient condition seems to be necessary for a large class of data: for example, it is already necessary in the case of a constant function $\lambda$ when the function $\gamma$ is genuinely nonlinear.

This paper is organized as follows:

- Section 2 is devoted to a steady version of (1.1): a short review of the concept of $\mathrm{H}$-convergence is made, some examples of application of this notion are given; then results are recalled on finite volume methods for elliptic problems, the notion of $\mathrm{H}$ convergence is then adapted to the study of the convergence of numerical schemes for elliptic problems and the convergence study of the coupled scheme for the twophase flow problem is completed (the complete proofs of the results stated in this section can be found in [8]).

- The transient problem is studied in Section 3.

- Some concluding remarks give the place of this work within on-going research. 


\section{The steady case.}

2.1. Some results of $\mathbf{H}$-convergence for the elliptic case. Let us first give the notations for the continuous Dirichlet problem. Let $\Omega$ be an open bounded subset of $\mathbb{R}^{N}$, with $N \in \mathbb{N}_{*}$ and let $\alpha$ and $\beta$ be two real numbers, with $0<\alpha \leq \beta$. We denote by $\mathcal{M}(\alpha, \beta, \Omega)$ the set of measurable functions $M: \Omega \longrightarrow \mathcal{L}\left(\mathbb{R}^{N}, \mathbb{R}^{N}\right)$ such that, for a.e. $x \in \Omega$ and for all $(\xi, \chi) \in\left(\mathbb{R}^{N}\right)^{2}, \alpha|\xi|^{2} \leq M(x) \xi \cdot \xi \leq \beta|\xi|^{2}$, and $M(x) \xi \cdot \chi=\xi \cdot M(x) \chi$. In the particular case where there exists a function $\mu \in L^{\infty}(\Omega)$ such that, for a.e. $x \in \Omega$, $M(x)=\mu(x) I_{N}$, where $I_{N}$ denotes the identity application from $\mathbb{R}^{N}$ to $\mathbb{R}^{N}$, we then denote $M=\mu$. In this case, we say that $M$ represents an isotropic field; otherwise, we say that the field $M$ is anisotropic. For a given source term $b \in H^{-1}(\Omega)$ and a given $M \in \mathcal{M}(\alpha, \beta, \Omega)$, we denote by $\mathcal{F}(b, M)$ the unique solution $\bar{u}$ of

$$
\bar{u} \in H_{0}^{1}(\Omega) \text { and } \int_{\Omega} M(x) \nabla \bar{u}(x) \cdot \nabla \bar{v}(x) d x=b(\bar{v}), \forall \bar{v} \in H_{0}^{1}(\Omega) .
$$

The following result, given in [17] (in which it was called G-convergence, in reference to some de Giorgi's works), has been extended in [19] to some more general configurations.

ThEOREM 1 (H-convergence). Let $\Omega$ be an open bounded subset of $\mathbb{R}^{N}$, with $N \in \mathbb{N}_{*}$. Let two real numbers $\alpha$ and $\beta$ be such that $0<\alpha \leq \beta$. Let $\left(M_{n}\right)_{n \in \mathbb{N}}$ be a sequence of elements of $M_{n} \in \mathcal{M}(\alpha, \beta, \Omega)$.

Then there exists a subsequence of $\left(M_{n}\right)_{n \in \mathbb{N}}$, again denoted $\left(M_{n}\right)_{n \in \mathbb{N}}$, and a function $M \in \mathcal{M}(\alpha, \beta, \Omega)$ such that:

- for all $b \in H^{-1}(\Omega), \mathcal{F}\left(b, M_{n}\right)$ weakly converges to $\mathcal{F}(b, M)$ in $H_{0}^{1}(\Omega)$ as $n \longrightarrow \infty$,

- for all $b \in H^{-1}(\Omega), M_{n} \nabla \mathcal{F}\left(b, M_{n}\right)$ weakly converges to $M \nabla \mathcal{F}(b, M)$ in $\left(L^{2}(\Omega)\right)^{N}$ as $n \longrightarrow \infty$.

We then say that the sequence $\left(M_{n}\right)_{n \in \mathbb{N}} H$-converges to $M$, called the H-limit of the sequence.

We now give some examples of H-convergence results. Let us first handle the onedimensional case. Let us suppose that $\Omega=(0,1)$. The sequence $\left(M_{n}\right)_{n \in \mathbb{N}}$ such that for all $n \in \mathbb{N}, M_{n} \in \mathcal{M}(\alpha, \beta, \Omega)$ is then a sequence of functions belonging to $L^{\infty}(\Omega)$ and $1 / M_{n}(x) \in[1 / \beta, 1 / \alpha]$ for a.e. $x \in \Omega$. For a given $f \in L^{2}(\Omega)$, we denote by $\hat{f}$ the continuous function defined, for all $x \in(0,1)$, by $\hat{f}(x)=\int_{(0, x)} f(s) d s$. We then have, for all $x \in \Omega$,

$$
\mathcal{F}\left(f, M_{n}\right)(x)=\frac{\int_{(0, x)}\left(1 / M_{n}(t)\right) d t}{\int_{(0,1)}\left(1 / M_{n}(t)\right) d t} \int_{(0,1)} \frac{\hat{f}(t)}{M_{n}(t)} d t-\int_{(0, x)} \frac{\hat{f}(t)}{M_{n}(t)} d t .
$$

Up to a subsequence, we can suppose that the sequence $\left(1 / M_{n}\right)_{n \in \mathbb{N}}$ converges to a function $1 / M$ for the weak $\star$ topology of $L^{\infty}(\Omega)$. We then get that, for all $x \in \Omega$,

$$
\lim _{n \longrightarrow \infty} \mathcal{F}\left(f, M_{n}\right)(x)=\frac{\int_{(0, x)}(1 / M(t)) d t}{\int_{(0,1)}(1 / M(t)) d t} \int_{(0,1)} \frac{\hat{f}(t)}{M(t)} d t-\int_{(0, x)} \frac{\hat{f}(t)}{M(t)} d t,
$$

which proves that $M$ is the $\mathrm{H}$-limit of this subsequence. Unfortunately, such a relation between the limit for the weak $\star$ topology of $L^{\infty}(\Omega)$ and the H-limit cannot be obtained in the general case $N>1$.

Some examples can now be given in two space dimensions. Let $\mu_{r}>0$ and $\mu_{b}>0$ be two real values respectively defining the permeability of two materials, respectively called "red" and "black". We first define the so-called checkerboard problem, setting $M_{1}: \mathbb{R}^{2} \longrightarrow \mathbb{R}$ by $\left(x_{1}, x_{2}\right) \mapsto \mu_{r}$ if $\operatorname{Int}\left(x_{1}\right)+\operatorname{Int}\left(x_{2}\right) \in 2 \mathbb{Z}$ (denoting for all $z \in \mathbb{R}$ by $\operatorname{Int}(z)$ the largest relative integer value lower than $z$ ), else $\left(x_{1}, x_{2}\right) \mapsto \mu_{b}$ (for example, $\operatorname{Int}(0.5)+\operatorname{Int}(0.5)=0$ and $M_{1}(0.5,0.5)=\mu_{r}$, Int $(1.5)+\operatorname{Int}(-1.5)=1-2=-1$ and $M_{1}(1.5,-1.5)=\mu_{b}$ : see Figure 2.1). Then we define, for all $n \in \mathbb{N}_{*}, M_{n}: \mathbb{R}^{2} \longrightarrow \mathbb{R}$ by $M_{n}\left(x_{1}, x_{2}\right)=M_{1}\left(n x_{1}, n x_{2}\right)$. It can then be shown that, in all open domain $\Omega$ of $\mathbb{R}^{2}$, the sequence $\left(M_{n}\right)_{n \in \mathbb{N}}$ H-converges to the constant function $\left(x_{1}, x_{2}\right) \mapsto \sqrt{\mu_{r} \mu_{b}}$. In this case, the H-limit of a sequence of isotropic heterogeneous fields is an isotropic homogeneous field. 

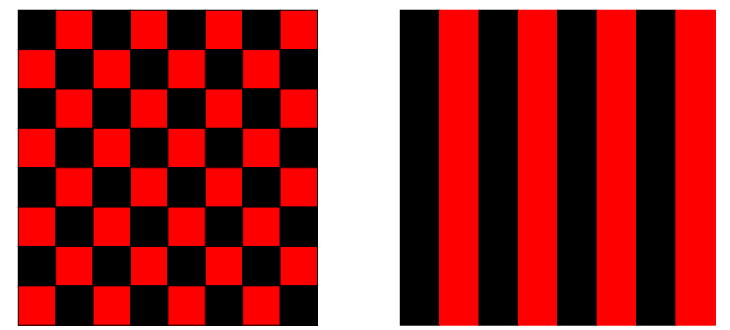

FIG. 2.1. The checkerboard case (left) and the multilayer case(right)

Another example involving two materials is the multilayer case, obtained with defining $M_{1}: \mathbb{R}^{2} \longrightarrow \mathbb{R}$ by $\left(x_{1}, x_{2}\right) \mapsto \mu_{r}$ if $\operatorname{Int}\left(x_{1}\right) \in 2 \mathbb{Z}$, else $\left(x_{1}, x_{2}\right) \mapsto \mu_{b}$ (for example, $\operatorname{Int}(0.5)=0$ and $M_{1}(0.5,10)=\mu_{r}, \operatorname{Int}(1.5)=1$ and $M_{1}(1.5,-4)=\mu_{b}$ : see Figure 2.1). We again define the sequence $\left(M_{n}\right)_{n \in \mathbb{N}}$, by $M_{n}: \mathbb{R}^{2} \longrightarrow \mathbb{R},\left(x_{1}, x_{2}\right) \mapsto M_{1}\left(n x_{1}, n x_{2}\right)$, for all $n \in \mathbb{N}_{*}$. Then it can be proved that the sequence $\left(M_{n}\right)_{n \in \mathbb{N}} \mathrm{H}$-converges, in all open domain $\Omega$ of $\mathbb{R}^{2}$, to the constant field, the value of which is the linear function defined by $(1,0) \mapsto\left(\frac{2 \mu_{r} \mu_{b}}{\mu_{r}+\mu_{b}}, 0\right)$ and $(0,1) \mapsto\left(0, \frac{\mu_{r}+\mu_{b}}{2}\right)$. We can remark that $\frac{2 \mu_{r} \mu_{b}}{\mu_{r}+\mu_{b}}$ is the harmonic average of $\mu_{r}$ and $\mu_{b}$, that is the invert of the average value of the inverts of $\mu_{r}$ and $\mu_{b}$ (this is exactly the value obtained by $\mathrm{H}$-convergence in the one-dimensional case), whereas $\frac{\mu_{r}+\mu_{b}}{2}$ is the arithmetic average of $\mu_{r}$ and $\mu_{b}$. In this two-dimensional case, the H-limit of a sequence of isotropic heterogeneous fields is an anisotropic homogeneous field.

Note that in the two above examples, the limit of $\left(M_{n}\right)_{n \in \mathbb{N}}$ for the weak $\star$ topology of $L^{\infty}(\Omega)$ is the constant function $\left(x_{1}, x_{2}\right) \mapsto \frac{\mu_{r}+\mu_{b}}{2}$. Using the notion of nonlinear weak $\star$ convergence (see [9]), the limit of $\left(M_{n}\right)_{n \in \mathbb{N}}$ in terms of Young's measure is the constant field of probability measure $\frac{1}{2} \delta_{\mu_{r}}+\frac{1}{2} \delta_{\mu_{b}}$, equivalently given by the function $\mu \in L^{\infty}(\Omega \times(0,1))$ such that, for a.e. $x \in \Omega$ and $s \in\left(0, \frac{1}{2}\right), \mu(x, s)=\mu_{r}$ and for a.e. $x \in \Omega$ and $s \in\left(\frac{1}{2}, 1\right)$, $\mu(x, s)=\mu_{b}$. Thus we see that the notion of nonlinear weak $\star$ convergence does not account for the heterogeneous spatial structure, and justifies the attempts of finding some more suitable generalized limit (see for example [20]).

2.2. Finite Volume meshes and schemes. We first recall the notion of admissible discretization [9] which is used in the definition of the finite volume scheme.

DeFinition 1 (Admissible discretization). Let $\Omega$ be an open bounded polygonal subset of $\mathbb{R}^{N}$, with $N \in \mathbb{N}_{*}$ (in general, we have $N=2$ or $N=3$ ). We denote $\partial \Omega=\bar{\Omega} \backslash \Omega$. An admissible finite volume discretization of $\Omega$, denoted by $\mathcal{D}$, is given by $\mathcal{D}=(\mathcal{T}, \mathcal{E}, \mathcal{P})$, where:

- $\mathcal{T}$ is a finite family of non empty open polygonal convex disjoint subsets of $\Omega$ (the "control volumes") such that $\bar{\Omega}=\cup_{K \in \mathcal{T}} \bar{K}$. We then denote, for all $K \in \mathcal{T}$, by $\partial K=\bar{K} \backslash K$ the boundary of $K$ and $m_{K}>0$ the $N$-dimensional Lebesgue measure of $K$ (it is the area of $K$ in the two-dimensional case and the volume in the threedimensional case).

- $\mathcal{E}$ is a finite family of disjoint subsets of $\bar{\Omega}$ (the "edges" of the mesh), such that, for all $\sigma \in \mathcal{E}$, there exists a hyperplane $E$ of $\mathbb{R}^{N}$ and $K \in \mathcal{T}$ with $\bar{\sigma}=\partial K \cap E$ and $\sigma$ is a non empty open subset of $E$. We then denote $m_{\sigma}>0$ the $(N-1)$-dimensional measure of $\sigma$. We assume that, for all $K \in \mathcal{T}$, there exists a subset $\mathcal{E}_{K}$ of $\mathcal{E}$ such that $\partial K=\cup_{\sigma \in \mathcal{E}_{K}} \bar{\sigma}$. It then results from the previous hypotheses that, for all $\sigma \in \mathcal{E}$, either $\sigma \subset \partial \Omega$ or there exists $(K, L) \in \mathcal{T}^{2}$ with $K \neq L$ such that $\bar{K} \cap \bar{L}=\bar{\sigma}$; we denote in the latter case $\sigma=K \mid L$.

- $\mathcal{P}$ is a family of points of $\Omega$ indexed by $\mathcal{T}$, denoted by $\mathcal{P}=\left(x_{K}\right)_{K \in \mathcal{T}}$. This family is such that, for all $K \in \mathcal{T}, x_{K} \in K$. For all $\sigma \in \mathcal{E}$ such that there exists $(K, L) \in \mathcal{T}^{2}$ with $\sigma=K \mid L$, it is assumed that the straight line $\left(x_{K}, x_{L}\right)$ going through $x_{K}$ and 
$x_{L}$ is orthogonal to $K \mid L$. For all $K \in \mathcal{T}$ and all $\sigma \in \mathcal{E}_{K}$, let $y_{\sigma}$ be the orthogonal projection of $x_{K}$ on $\sigma$. We suppose that $y_{\sigma} \in \sigma$.

The following notations are used. The size of the discretization is defined by:

$$
\operatorname{size}(\mathcal{D})=\sup \{\operatorname{diam}(K), K \in \mathcal{T}\} .
$$

For all $K \in \mathcal{T}$ and $\sigma \in \mathcal{E}_{K}$, we denote by $\mathbf{n}_{K, \sigma}$ the unit vector normal to $\sigma$ outward to $K$. We define a subset of $K$ associated with the edge $\sigma$ by

$$
D_{K, \sigma}=\left\{t x_{K}+(1-t) y, t \in(0,1), y \in \sigma\right\}
$$

(the letter "D" stands for "diamond") and denote by $d_{K, \sigma}$ the euclidean distance between $x_{K}$ and $\sigma$. We then define

$$
\tau_{K, \sigma}=\frac{m_{\sigma}}{d_{K, \sigma}} .
$$

The set of interior (resp. boundary) edges is denoted by $\mathcal{E}_{\text {int }}$ (resp. $\mathcal{E}_{\text {ext }}$ ), that is $\mathcal{E}_{\text {int }}=\{\sigma \in \mathcal{E} ; \sigma \not \subset \partial \Omega\}$ (resp. $\mathcal{E}_{\text {ext }}=\{\sigma \in \mathcal{E} ; \sigma \subset \partial \Omega\}$ ).

Some discrete spaces of functions are then defined.

Definition 2. Let $\Omega$ be an open bounded polygonal subset of $\mathbb{R}^{N}$, with $N \in \mathbb{N}_{*}$. Let $\mathcal{D}=(\mathcal{T}, \mathcal{E}, \mathcal{P})$ be an admissible finite volume discretization of $\Omega$ in the sense of Definition 1. We denote by $H_{\mathcal{D}}(\Omega) \subset L^{2}(\Omega)$ the space of functions which admit a constant value in each $K \in \mathcal{T}$. For all $u \in H_{\mathcal{D}}(\Omega)$ and for all $K \in \mathcal{T}$, we denote by $u_{K}$ the constant value of $u$ in $K$ and we define $\left(u_{\sigma}\right)_{\sigma \in \mathcal{E}}$ by

and

$$
u_{\sigma}=0, \forall \sigma \in \mathcal{E}_{\text {ext }}
$$

$$
\tau_{K, \sigma}\left(u_{\sigma}-u_{K}\right)+\tau_{L, \sigma}\left(u_{\sigma}-u_{L}\right)=0, \forall \sigma \in \mathcal{E}_{\text {int }}, \sigma=K \mid L .
$$

We now give a definition of an approximate gradient of the functions of $H_{\mathcal{D}}(\Omega)$. We define a function, denoted $\mathbf{G}_{\mathcal{D}}: H_{\mathcal{D}}(\Omega) \longrightarrow\left(L^{2}(\Omega)\right)^{N}, u \longrightarrow \mathbf{G}_{\mathcal{D}} u$ with

$$
\mathbf{G}_{\mathcal{D}} u(x)=\frac{N}{d_{K, \sigma}}\left(u_{\sigma}-u_{K}\right) \mathbf{n}_{K, \sigma}, \text { for a.e. } x \in D_{K, \sigma}, \forall K \in \mathcal{T}, \forall \sigma \in \mathcal{E}_{K} .
$$

Let two real numbers $\alpha$ and $\beta$ be such that $0<\alpha \leq \beta$. We denote by $\mathcal{M}_{\mathcal{D}}(\alpha, \beta) \subset$ $L^{\infty}(\Omega)$ the set of functions $\mu$ such that for all $\sigma \in \mathcal{E}$, there exists a constant value, denoted $\mu_{\sigma} \in[\alpha, \beta]$, such that

$$
\mu(x)=\mu_{\sigma}, \forall x \in D_{K, \sigma} \text { where } K \text { is such that } \sigma \in \mathcal{E}_{K} .
$$

The function which takes the constant value 1 on $\Omega$ is denoted by 1 . For $(u, v) \in$ $\left(H_{\mathcal{D}}(\Omega)\right)^{2}$ and $\varphi \in C^{0}(\bar{\Omega})$, we denote by

$$
[u, v]_{\mathcal{D}, \mu, \varphi}=\sum_{K \in \mathcal{T}} \varphi\left(x_{K}\right) \sum_{\sigma \in \mathcal{E}_{K}} \mu_{\sigma} \tau_{K, \sigma}\left(u_{\sigma}-u_{K}\right)\left(v_{\sigma}-v_{K}\right) .
$$
by

We define the following norm in $H_{\mathcal{D}}(\Omega)$ using the discrete Poincaré inequality (see [9])

$$
|u|_{\mathcal{D}}=\left([u, u]_{\mathcal{D}, 1,1}\right)^{1 / 2} .
$$

REMARK 1. For all edge $\sigma$ such that $\sigma=K \mid L$, the function $\mathbf{G}_{\mathcal{D}} u$ is constant on $D_{K, \sigma} \cup D_{L, \sigma}$.

We now give a finite volume scheme for a Dirichlet problem on $\Omega$. Let $\mathcal{D}=(\mathcal{T}, \mathcal{E}, \mathcal{P})$ be an admissible discretization of $\Omega$ in the sense of Definition 1. Let two real numbers $\alpha$ and $\beta$ be such that $0<\alpha \leq \beta$ and let $\mu \in \mathcal{M}_{\mathcal{D}}(\alpha, \beta)$. For a given $f \in L^{2}(\Omega)$, let $u \in H_{\mathcal{D}}(\Omega)$ (cf Definition 2) be such that 


$$
-\sum_{\sigma \in \mathcal{E}_{K}} \mu_{\sigma} \tau_{K, \sigma}\left(u_{\sigma}-u_{K}\right)=\int_{K} f(x) d x, \forall K \in \mathcal{T}
$$

(the existence and uniqueness of $u \in H_{\mathcal{D}}(\Omega)$ solution of (2.5) results from the inequality $\mu_{\sigma} \geq \alpha$ for all $\sigma \in \mathcal{E}$ and from the discrete Poincaré inequality, see [9]). Since, for all $v \in H_{\mathcal{D}}(\Omega), \sum_{K \in \mathcal{T}} \sum_{\sigma \in \mathcal{E}_{K}} \mu_{\sigma} \tau_{K, \sigma}\left(u_{\sigma}-u_{K}\right) v_{\sigma}=0$ thanks to $(2.1)-(2.2),(2.5)$ is equivalent to

$$
u \in H_{\mathcal{D}}(\Omega) \text { and }[u, v]_{\mathcal{D}, \mu, 1}=\int_{\Omega} f(x) v(x) d x, \forall v \in H_{\mathcal{D}}(\Omega) .
$$

We then have the following results, proven in [9] and in [8].

LEMMA 2.1 (Finite volume method). Let $\Omega$ be an open bounded polygonal subset of $\mathbb{R}^{N}$, with $N \in \mathbb{N}_{*}$. Let $\mathcal{D}=(\mathcal{T}, \mathcal{E}, \mathcal{P})$ be an admissible finite volume discretization of $\Omega$ in the sense of Definition 1. Let two real numbers $\alpha$ and $\beta$ be such that $0<\alpha \leq \beta$ and let $\mu \in \mathcal{M}_{\mathcal{D}}(\alpha, \beta)$. Let $f \in L^{2}(\Omega)$.

Then there exists one and only one $u \in H_{\mathcal{D}}(\Omega)$ (cf Definition 2) given by (2.5). We then denote $u=F_{\mathcal{D}}(f, \mu)$. Moreover,

$$
\alpha|u|_{\mathcal{D}} \leq \operatorname{diam}(\Omega)\|f\|_{L^{2}(\Omega)} .
$$

In the case $\mu=1$, we have the following convergence results: $F_{\mathcal{D}}(f, 1)$ converges to $\mathcal{F}(f, 1)$ in $L^{2}(\Omega)$ as $\operatorname{size}(\mathcal{D}) \longrightarrow 0, \mathbf{G}_{\mathcal{D}} F_{\mathcal{D}}(f, 1)$ weakly converges to $\nabla \mathcal{F}(f, 1)$ as $\operatorname{size}(\mathcal{D}) \longrightarrow 0$ in $\left(L^{2}(\Omega)\right)^{N}$, and

$$
\int_{\Omega} \varphi(x)(\nabla \mathcal{F}(f, 1)(x))^{2} d x=\lim _{\operatorname{size}(\mathcal{D}) \longrightarrow 0}\left[F_{\mathcal{D}}(f, 1), F_{\mathcal{D}}(f, 1)\right]_{\mathcal{D}, 1, \varphi}, \quad \forall \varphi \in C^{0}(\bar{\Omega}) .
$$

2.3. The Hd-convergence theorem and relations with $\mathbf{H}$-convergence. The following theorem (proven in [8]) expresses a discrete version of Theorem 1.

THEOREM 2 (Hd-convergence). Let $\Omega$ be an open bounded polygonal subset of $\mathbb{R}^{N}$, with $N \in \mathbb{N}_{*}$. Let two real numbers $\alpha$ and $\beta$ be such that $0<\alpha \leq \beta$. Let $\left(\mathcal{D}_{n}, \mu_{n}\right)_{n \in \mathbb{N}}$ be a sequence such that, for all $n \in \mathbb{N}, \mathcal{D}_{n}$ is an admissible discretization of $\Omega$ in the sense of Definition 1, and $\mu_{n} \in \mathcal{M}_{\mathcal{D}_{n}}(\alpha, \beta)$. We assume that $\lim _{n \rightarrow \infty} \operatorname{size}\left(\mathcal{D}_{n}\right)=0$.

Then there exist a subsequence of $\left(\mathcal{D}_{n}, \mu_{n}\right)_{n \in \mathbb{N}}$, again denoted $\left(\mathcal{D}_{n}, \mu_{n}\right)_{n \in \mathbb{N}}$, and a unique measurable function $M \in \mathcal{M}(\alpha, \beta, \Omega)$ (this set is defined in Section 2.1) such that:

- for all $f \in L^{2}(\Omega), F_{\mathcal{D}_{n}}\left(f, \mu_{n}\right)$ converges to $\mathcal{F}(f, M)$ in $L^{2}(\Omega)$ as $n \longrightarrow \infty$ and $\mathbf{G}_{\mathcal{D}_{n}} F_{\mathcal{D}_{n}}\left(f, \mu_{n}\right)$ weakly converges to $\nabla \mathcal{F}(f, M)$ in $\left(L^{2}(\Omega)\right)^{N}$ as $n \longrightarrow \infty$ (the functions $F_{\mathcal{D}}(f, \mu)$, denoting the discrete solution of a finite volume scheme for an elliptic problem with the homogeneous Dirichlet boundary condition, the right hand side $f$ and a discrete diffusion field $\mu$, and $\mathbf{G}_{\mathcal{D}} F_{\mathcal{D}}(f, \mu)$, denoting a discrete gradient of this numerical solution, are defined in Section 2.2 and the function $\mathcal{F}(f, M)$, denoting the solution of an elliptic problem with the homogeneous Dirichlet boundary condition, the right hand side $f$ and a diffusion matrix field $M$, is defined in Section 2.1),

- for all $f \in L^{2}(\Omega), \mu_{n} \mathbf{G}_{\mathcal{D}_{n}} F_{\mathcal{D}_{n}}\left(f, \mu_{n}\right)$ weakly converges to $M \nabla \mathcal{F}(f, M)$ in $\left(L^{2}(\Omega)\right)^{N}$ as $n \longrightarrow \infty$.

We then say that the sequence $\left(\mathcal{D}_{n}, \mu_{n}\right)_{n \in \mathbb{N}} H d$-converges to $M$, called the Hd-limit of the sequence.

Some comments can be done on the relation between Hd-convergence and H-convergence. Let us first study the one-dimensional case. We take again the above notations for the onedimensional case. Let $\Omega=(0,1), \alpha$ and $\beta$ be such that $0<\alpha \leq \beta$. In order to define an admissible discretization of $\Omega$, let $p \in \mathbb{N}_{*}$ and let $\left(y_{k}\right)_{k=0, \ldots, p}$ and $\left(x_{k}\right)_{k=1, \ldots, p}$ be real values such that 


$$
y_{0}=0<x_{1}<y_{1}<x_{2} \ldots<y_{k-1}<x_{k}<y_{k} \ldots<y_{p-1}<x_{p}<y_{p}=1 .
$$

Then the discretization $\mathcal{D}=(\mathcal{T}, \mathcal{E}, \mathcal{P})$ defined by $\mathcal{T}=\left\{\left(y_{k-1}, y_{k}\right), k=1, \ldots, p\right\}, \mathcal{E}=$ $\left\{\left\{y_{k}\right\}, k=0, \ldots, p\right\}$ and $\mathcal{P}=\left\{x_{k}, k=1, \ldots, p\right\}$ is an admissible discretization of $\Omega$ in the sense of Definition 1. Let $f \in L^{2}(\Omega)$ and $\mu \in \mathcal{M}_{\mathcal{D}}(\alpha, \beta)$ be given (recall that the function $\mu$ takes constant values in $\left.\left(0, x_{1}\right), \ldots,\left(x_{k}, x_{k+1}\right), \ldots,\left(x_{p}, 1\right)\right)$. We again define the function $\hat{f}$ by $\hat{f}(x)=\int_{(0, x)} f(t) d t$ for all $x \in \Omega$, and we introduce the function $\hat{f}_{\mathcal{D}}$ defined, by $\hat{f}_{\mathcal{D}}(x)=0=\hat{f}\left(y_{0}\right)$ for all $x \in\left(0, x_{1}\right)$, by $\hat{f}_{\mathcal{D}}(x)=\hat{f}\left(y_{k}\right)$ for all $x \in\left(x_{k}, x_{k+1}\right)$, and by $\hat{f}_{\mathcal{D}}(x)=\hat{f}(1)$ for all $x \in\left(x_{p}, 1\right)$. Some calculations show that the solution of the finite volume scheme (2.5) is defined by

$$
\begin{aligned}
& F_{\mathcal{D}}(f, \mu)(x)=\frac{\int_{\left(0, x_{k}\right)}(1 / \mu(t)) d t}{\int_{(0,1)}(1 / \mu(t)) d t} \int_{(0,1)} \frac{\hat{f}_{\mathcal{D}}(t)}{\mu(t)} d t-\int_{\left(0, x_{k}\right)} \frac{\hat{f}_{\mathcal{D}}(t)}{\mu(t)} d t \\
& \forall x \in\left(y_{k-1}, y_{k}\right), \quad \forall k=1, \ldots, p .
\end{aligned}
$$

Let $\left(\mathcal{D}_{n}, \mu_{n}\right)_{n \in \mathbb{N}}$ be a sequence such that, for all $n \in \mathbb{N}, \mathcal{D}_{n}$ is an admissible discretization of $\Omega$ in the sense of Definition 1 , and $\mu_{n} \in \mathcal{M}_{\mathcal{D}_{n}}(\alpha, \beta)$. We assume that $\lim _{n \rightarrow \infty} \operatorname{size}\left(\mathcal{D}_{n}\right)=0$. Up to a subsequence, we can suppose that the sequence $\left(1 / \mu_{n}\right)_{n \in \mathbb{N}}$ converges to a function $1 / M$ for the weak $\star$ topology of $L^{\infty}(\Omega)$. Since the sequence $\left(\hat{f}_{\mathcal{D}_{n}}\right)_{n \in \mathbb{N}}$ strongly converges to the continuous function $\hat{f}$ as $n \longrightarrow \infty$, we get, using (2.9) in which we let $\mathcal{D}=\mathcal{D}_{n}$ and $\mu=\mu_{n}$, that the limit of the sequence $\left(F_{\mathcal{D}_{n}}\left(f, \mu_{n}\right)\right)_{n \in \mathbb{N}}$ is exactly the function $\mathcal{F}(f, M)$ defined, for all $x \in \Omega$, by

$$
\mathcal{F}(f, M)(x)=\frac{\int_{(0, x)}(1 / M(t)) d t}{\int_{(0,1)}(1 / M(t)) d t} \int_{(0,1)} \frac{\hat{f}(t)}{M(t)} d t-\int_{(0, x)} \frac{\hat{f}(t)}{M(t)} d t .
$$

This proves that the Hd-limit of $\left(\mathcal{D}_{n}, \mu_{n}\right)_{n \in \mathbb{N}}$ is the function $M$, and therefore coincides, when using the finite volume scheme $(2.5)$, with the H-limit of $\left(\mu_{n}\right)_{n \in \mathbb{N}}$; the use of some convergence for the weak $\star$ topology of $L^{\infty}(\Omega)$ is again sufficient to pass to the limit.

REMARK 2. Note that the coincidence of the discrete and the continuous H-limits is not true for all the one-dimensional numerical schemes which can be associated with the same function $\mu$. Indeed, assume, in order to simplify, that $y_{k}-y_{k-1}=h$, for $k=1, \ldots, p$ (with $h=1 / p), x_{k}=\left(y_{k}+y_{k-1}\right) / 2$, for $k=1, \ldots, p$, and that the function $\mu$ takes constant values in $\left(0, x_{1}\right), \ldots,\left(x_{k}, x_{k+1}\right), \ldots,\left(x_{p}, 1\right)$ which are $\mu_{r}$ and $\mu_{b}$ in alternance. If we discretize the Dirichlet problem with this function $\mu$ as diffusion coefficient and the piecewise linear finite element scheme with nodes located at the points $\left(y_{k}\right)_{k=0, \ldots, p}$, we obtain an approximate solution which is exactly the same as the one which is obtained by the same method (piecewise linear finite element) and a constant value of $\mu$ as diffusion coefficient, namely the arithmetic average of $\mu_{r}$ and $\mu_{b}$. Then, this approximate solution converges, as $h \longrightarrow 0$, towards the solution of the Dirichlet problem whose diffusion is this arithmetic average of $\mu_{r}$ and $\mu_{b}$, whereas the H-limit as $h \longrightarrow 0$ of the continuous operators is given by the harmonic average of $\mu_{r}$ and $\mu_{b}$.

However, in the case $N>1$ and even in the isotropic case, passing to the limit for the weak $\star$ topology of $L^{\infty}(\Omega)$ does not deliver the Hd-limit. Indeed, let us consider the sequence of admissible discretizations $\mathcal{D}_{n}$ of $\Omega=(0,1) \times(0,1)$, where the control volumes are some $(k / n,(k+1) / n) \times(l / n,(l+1) / n)$, for integer values $k$ and $l$ between 0 and $n-1$ (see Figure $2.2)$. Assume that the function $\mu_{n}$ is defined by the value $\mu_{r}>0$ on the vertical edges $\{k / n\} \times$ $(l / n,(l+1) / n)$ and by the value $\mu_{b}>0$ on the horizontal edges $(k / n,(k+1) / n) \times\{l / n\}$. Then the function $\mu_{n} \in \mathcal{M}_{\mathcal{D}_{n}}(\alpha, \beta)$ (with $\alpha=\min \left(\mu_{r}, \mu_{b}\right)$ and $\beta=\max \left(\mu_{r}, \mu_{b}\right)$ ) corresponds to the first case of the above two-dimensional examples in Section 2.1 (recall that the function $\mu_{n}$ is constant on subsets which, in this case, are the squares the side of which has its length equal to $1 /(n \sqrt{2})$, tilted with an angle of measure $\pi / 4$ with respect to the grid. As seen above, the H-limit of $\left(\mu_{n}\right)_{n \in \mathbb{N}}$ is the field with constant value $\sqrt{\mu_{r} \mu_{b}}$. We then remark that for a given $f \in L^{2}(\Omega)$, the discrete values solution of the finite volume scheme (2.5) are identical 
to those obtained from $(2.5)$, written in the case where $\tilde{\Omega}=\left(0,1 / \sqrt{\mu_{r}}\right) \times\left(0,1 / \sqrt{\mu_{b}}\right)$, the grid is given by the subsets $\left(k /\left(n \sqrt{\mu_{r}}\right),(k+1) /\left(n \sqrt{\mu_{r}}\right)\right) \times\left(l /\left(n \sqrt{\mu_{b}}\right),(l+1) /\left(n \sqrt{\mu_{b}}\right)\right), \mu=1$, and the right hand side $\tilde{f}=f\left(\cdot \sqrt{\mu_{r}}, \cdot \sqrt{\mu_{b}}\right)$. Thanks to Lemma 2.1 which states the convergence of the finite volume scheme for $\mu=1$ we then get that $u_{\mathcal{D}_{n}}$ converges to $\bar{u}=\tilde{u}\left(\cdot / \sqrt{\mu_{r}}, \cdot / \sqrt{\mu_{b}}\right)$ with $\tilde{u}=\mathcal{F}_{\tilde{\Omega}}(\tilde{f}, 1)$, denoting here by $\mathcal{F}_{\tilde{\Omega}}$ the function $\mathcal{F}$ obtained when the Dirichlet problem is solved in the domain $\tilde{\Omega}$. An easy change of variable proves that $\bar{u}=\mathcal{F}(f, M)$, where $M$ is the constant field, the value of which is the linear application defined by $(1,0) \mapsto\left(\mu_{r}, 0\right)$ and $(0,1) \mapsto\left(0, \mu_{b}\right)$. This field $M$, which is homogeneous anisotropic and differs from the H-limit of $\left(\mu_{n}\right)_{n \in \mathbb{N}}$, is therefore the Hd-limit of $\left(\mathcal{D}_{n}, \mu_{n}\right)_{n \in \mathbb{N}}$.

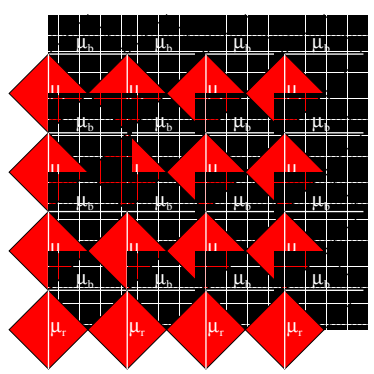

FIG. 2.2. Case of discrepancy between the $H$ - and Hd-limits

The physical reason of this discrepancy is the fact that in this example, the heterogeneous behavior and the grid are at the same scale: note that this occurs when solving the coupled two-phase flow in porous media problem using a coupled scheme on the same grid (see Section 2.4). On the contrary, in the cases where it is possible to let the size of the mesh tend to zero faster than the size of the heterogenities, the obtained $\mathrm{H}$ - and Hd-limits are equal.

REMARK 3. Similar results to Theorem 2 can be obtained within the finite element framework, leading to the same distinction between the resulting Hd-limit and the H-limit (see Remark 2 for an example in the one-dimensional case).

REMARK 4. Exactly in the same manner as for the continuous case, it is possible to show the local character of Hd-convergence in the sense of Theorem 2, and the independence of the Hd-limit on the boundary conditions (see [16] for such results within the finite difference setting).

The proof of Theorem 2 follows the following steps, similar to that which are used in the continuous setting:

1. we first prove that there exists a subsequence of $\left(\mathcal{D}_{n}, \mu_{n}\right)_{n \in \mathbb{N}}$, which we again denote by $\left(\mathcal{D}_{n}, \mu_{n}\right)_{n \in \mathbb{N}}$, that verifies: there exists an invertible continuous linear application $\bar{F}: H^{-1}(\Omega) \longrightarrow H_{0}^{1}(\Omega)$ and a continuous linear application $\overline{\mathbf{G}}: H^{-1}(\Omega) \longrightarrow$ $\left(L^{2}(\Omega)\right)^{N}$ such that, for all $f \in L^{2}(\Omega)$, the sequence $\left(F_{\mathcal{D}_{n}}\left(f, \mu_{n}\right)\right)_{n \in \mathbb{N}}$ converges to $\bar{F}(f)$ in $L^{2}(\Omega)$, the sequence $\left(\mathbf{G}_{\mathcal{D}_{n}} F_{\mathcal{D}_{n}}\left(f, \mu_{n}\right)\right)_{n \in \mathbb{N}}$ weakly converges to $\nabla \bar{F}(f)$ in $\left(L^{2}(\Omega)\right)^{N}$, and the sequence $\left(\mu_{n} \mathbf{G}_{\mathcal{D}_{n}} F_{\mathcal{D}_{n}}\left(f, \mu_{n}\right)\right)_{n \in \mathbb{N}}$ weakly converges to $\overline{\mathbf{G}}(f)$ in $\left(L^{2}(\Omega)\right)^{N}$.

2. We then prove a discrete version of the "div-curl" lemma, which allows to prove that $\overline{\mathbf{G}}(f) \cdot \nabla \bar{F}(g)=\overline{\mathbf{G}}(g) \cdot \nabla \bar{F}(f)$ a.e. in $\Omega$, for all $f$ and $g$.

3. The invertibility property of $\bar{F}$ leads to the conclusion, taking $g$ such that $\bar{F}(g)$ is locally a given constant vector.

2.4. Application to the steady coupled problem. We now study the convergence of the industrial scheme applied to the steady-state version of the evolution problem (1.1):

$$
\begin{array}{ll}
-\operatorname{div}(\lambda(s) \nabla u) & =\bar{f} \\
-\operatorname{div}(\gamma(s) \lambda(s) \nabla u) & =(\bar{f})^{+} \gamma(\bar{s})-(\bar{f})^{-} \gamma(s)
\end{array} \quad \text { in } \Omega,
$$


with the boundary conditions

$$
\begin{aligned}
& u=0 \text { on } \partial \Omega, \\
& s=\hat{s} \text { on }\left\{x \in \partial \Omega, \nabla u(x) \cdot \mathbf{n}_{\partial \Omega}(x) \geq 0\right\} .
\end{aligned}
$$

We refer to the introduction for the physical meaning of the quantities appearing in (2.10) and (2.11). The following assumptions (denoted in the following Hypotheses (H)) are made on the data:

- the domain $\Omega$ is an open polygonal connex subset of $\mathbb{R}^{N}$, with $N=2$ or $N=3$,

- $\gamma \in C^{0}([0,1],[0,1])$ is a nondecreasing Lipschitz continuous function with $\gamma(0)=0$ and $\gamma(1)=1$, and Lipschitz constant $L_{\gamma}>0$,

- there exists two real numbers $\alpha$ and $\beta$, with $0<\alpha \leq \beta$ such that $\lambda \in C^{0}([0,1],[\alpha, \beta])$ (recall that $\lambda$ is the "total mobility") verifies that $\gamma \lambda$ (the mobility of the phase 1 , also denoted below $k_{1}$ ) is nondecreasing and $(1-\gamma) \lambda$ (the mobility of the phase 2 ) is nonincreasing,

- $\bar{f} \in L^{2}(\Omega)$ represents the rates at the wells,

- $\bar{s} \in L^{\infty}(\Omega)$ is such that $0 \leq \bar{s} \leq 1$ a.e. in $\Omega$,

- $\hat{s} \in L^{\infty}(\partial \Omega)$ is such that $0 \leq \hat{s} \leq 1$ a.e. in $\partial \Omega$ (for the $N-1$ dimensional Lebesgue measure).

Let us write, under the above hypotheses, the industrial finite volume coupled scheme in this steady case. Let $\mathcal{D}$ be an admissible discretization of $\Omega$ in the sense of Definition 1 . We set

$$
\left.\begin{array}{ll}
\bar{f}_{K}=\int_{K} \bar{f}(x) d x, \bar{s}_{K}=\frac{1}{m_{K}} \int_{K} \bar{s}(x) d x, & \forall K \in \mathcal{T}, \\
\hat{s}_{\sigma}=\frac{1}{m_{\sigma}} \int_{\sigma} \hat{s}(x) d x, & \forall \sigma \in \mathcal{E}_{\text {ext }} .
\end{array}\right\}
$$

We introduce the set $L_{\mathcal{D}}(\Omega,[0,1])$ of the functions of $L^{\infty}(\Omega)$ whose value on each $K \in \mathcal{T}$ is a constant value belonging to $[0,1]$. For all $s \in L_{\mathcal{D}}(\Omega,[0,1])$ and $K \in \mathcal{T}$, we denote $s_{K} \in[0,1]$ the constant value of $s$ in $K$. For all $u \in H_{\mathcal{D}}(\Omega)$ and $s \in L_{\mathcal{D}}(\Omega,[0,1])$, the upstream evaluation of the saturation at the edges $\sigma \in \mathcal{E}$ is defined by the functions $s_{\sigma}(u, s, \hat{s})$ such that

$$
\begin{array}{llll}
s_{\sigma}(u, s, \hat{s}) & =s_{K} & \text { if } u_{K} \geq u_{L} & \\
s_{\sigma}(u, s, \hat{s}) & =s_{L} & \text { if } u_{K}<u_{L} & \\
s_{\sigma}(u, s, \hat{s}) & =s_{K} & \text { if } u_{K} \geq 0 \\
s_{\sigma}(u, s, \hat{s}) & =\hat{s}_{\sigma} & \text { if } u_{K}<0 & \forall \sigma \in \mathcal{E}_{\text {int }}, \sigma=K \mid L
\end{array}
$$

and the functions $\mu_{\sigma}(u, s, \hat{s}) \in \mathcal{M}_{\mathcal{D}}(\alpha, \beta)$ by

$$
\mu_{\sigma}(u, s, \hat{s})=\lambda\left(s_{\sigma}(u, s, \hat{s})\right), \forall \sigma \in \mathcal{E} .
$$

Then the following numerical scheme is used to build the approximation $(u, s) \in H_{\mathcal{D}}(\Omega) \times$ $L_{\mathcal{D}}(\Omega,[0,1])$ :

$$
\begin{gathered}
u=F(\bar{f}, \mathcal{D}, \mu(u, s, \hat{s})), \\
-\sum_{\sigma \in \mathcal{E}_{K}} \gamma\left(s_{\sigma}(u, s, \hat{s})\right) \mu_{\sigma}(u, s, \hat{s}) \tau_{K, \sigma}\left(u_{\sigma}-u_{K}\right)= \\
\gamma\left(\bar{s}_{K}\right)\left(\bar{f}_{K}\right)^{+}-\gamma\left(s_{K}\right)\left(\bar{f}_{K}\right)^{-}, \quad \forall K \in \mathcal{T} .
\end{gathered}
$$

REMARK 5. Note that the function $\lambda$ is also evaluated in (2.14) using an upstream weighted scheme, which corresponds to the fact that, in the industrial scheme, the mobilities of all the phases are upstream weighted. However, it would be natural to use a centered 
approximation in (2.14) and use an upstream weighted scheme for $\gamma$ in the left hand side of (2.16), but in such a case the convergence results given in Theorem 4 should be weakened.

Then the following lemma, stating the existence of a solution to the coupled scheme, can be proven (see [8]).

Lemma 2.2. Let us assume Hypotheses (H). Let $\mathcal{D}$ be an admissible discretization of $\Omega$ in the sense of Definition 1. Then there exists at least one solution $(u, s) \in H_{\mathcal{D}}(\Omega) \times L_{\mathcal{D}}(\Omega,[0,1])$ to Scheme (2.12)-(2.16).

The only convergence result that we are able to prove is the following (see [8]).

THEOREM 3. Let us assume Hypotheses $(H)$. Let $\left(\mathcal{D}_{n}\right)_{n \in \mathbb{N}}$ be a sequence such that, for all $n \in \mathbb{N}, \mathcal{D}_{n}$ is an admissible discretization of $\Omega$ in the sense of Definition 1 , and $\lim _{n \rightarrow \infty} \operatorname{size}\left(\mathcal{D}_{n}\right)=0$.

Then there exists a subsequence of $\left(\mathcal{D}_{n}\right)_{n \in \mathbb{N}}$, again denoted $\left(\mathcal{D}_{n}\right)_{n \in \mathbb{N}}$, such that, denoting for all $n \in \mathbb{N},\left(u_{n}, s_{n}, \mu_{n}\right) \in H_{\mathcal{D}_{n}}(\Omega) \times L_{\mathcal{D}_{n}}(\Omega,[0,1]) \times \mathcal{M}_{\mathcal{D}_{n}}(\alpha, \beta)$ the solution given by the scheme (2.12)-(2.16) with $\mathcal{D}=\mathcal{D}_{n}$, we have:

- the sequence $\left(\mathcal{D}_{n}, \mu_{n}\right)_{n \in \mathbb{N}} H d$-converges in the sense of Theorem 2 to a measurable function $M \in \mathcal{M}(\alpha, \beta, \Omega)$, which implies that $u_{n}$ converges to $\bar{u}=\mathcal{F}(\bar{f}, M)$ in $L^{2}(\Omega)$ as $n \longrightarrow \infty$,

- there exists a function $s \in L^{\infty}(\Omega)$, with $0 \leq s \leq 1$ a.e. such that the sequence $\left(s_{n}\right)_{n \in \mathbb{N}}$ converges to $s$ for the weak $\star$ topology of $L^{\infty}(\Omega)$ and there exists a function $\bar{\gamma} \in L^{\infty}(\Omega)$, with $0 \leq \bar{\gamma} \leq 1$ a.e. such that the sequence $\left(\gamma\left(s_{n}\right)\right)_{n \in \mathbb{N}}$ converges to $\bar{\gamma}$ for the weak $\star$ topology of $L^{\infty}(\Omega)$.

This result appears to be very weak compared to the initial ambition of approximating Problem (2.10). The first item of the conclusion of Theorem 3 is a direct consequence of Theorem 2. The second item is a consequence of the sequential weak $\star$ compactness of the closed balls of $L^{\infty}$. Note that, since the way to handle the convergence of (2.16) does not seem to be clear, no relation is given in the previous theorem between the limit of $\left(\gamma\left(s_{n}\right) \lambda\left(s_{n}\right)\right)_{n \in \mathbb{N}}$, which is a possibly degenerate diffusion if we consider the second equation of (2.10) as an elliptic equation on $u$, and the Hd-limit of $\left(\mathcal{D}_{n}, \mu_{n}\right)_{n \in \mathbb{N}}$. Such a relation can be found in the following particular case, where there exists a nondecreasing Lipschitz continuous function $k_{1}:[0,1] \longrightarrow \mathbb{R}$, with $k_{1}(0)=0$ and $k_{1}(1)>0$, and a real $\Lambda \in(0,1)$ such that:

$$
\begin{aligned}
\gamma(s) & =\frac{k_{1}(s)}{k_{1}(s)+\Lambda\left(k_{1}(1)-k_{1}(s)\right)}, \\
\lambda(s) & =k_{1}(s)+\Lambda\left(k_{1}(1)-k_{1}(s)\right), \quad \forall s \in[0,1] .
\end{aligned}
$$

Note that we can take in this case $\beta=k_{1}(1)$ and $\alpha=\Lambda k_{1}(1)$. This particular case corresponds to a mobility of the second phase defined by the function $\Lambda\left(k_{1}(1)-k_{1}().\right)$ (this can be acceptable in some physical situations; recall that $k_{1}$ is the mobility of the first phase). We can then give the following result, which is more complete than Theorem 3 (as previously mentioned, the following theorem does not give the limit of the scheme as a solution of (2.10) since we could only obtain such a result within a strong convergence property for $\left.\left(s_{n}\right)_{n \in \mathbb{N}}\right)$.

THEOREM 4. Let us assume Hypotheses $(H)$ in the particular case (2.17). Let $\left(\mathcal{D}_{n}\right)_{n \in \mathbb{N}}$ be a sequence such that, for all $n \in \mathbb{N}, \mathcal{D}_{n}$ is an admissible discretization of $\Omega$ in the sense of Definition 1, and $\lim _{n \rightarrow \infty} \operatorname{size}\left(\mathcal{D}_{n}\right)=0$.

Then there exists a subsequence of $\left(\mathcal{D}_{n}\right)_{n \in \mathbb{N}}$, again denoted $\left(\mathcal{D}_{n}\right)_{n \in \mathbb{N}}$ such that, denoting for all $n \in \mathbb{N},\left(u_{n}, s_{n}, \mu_{n}\right) \in H_{\mathcal{D}_{n}}(\Omega) \times L_{\mathcal{D}_{n}}(\Omega,[0,1]) \times \mathcal{M}_{\mathcal{D}_{n}}\left(\Lambda k_{1}(1), k_{1}(1)\right)$ the solution given by the scheme (2.12)-(2.16) with $\mathcal{D}=\mathcal{D}_{n}$, we have, in addition to the conclusions of Theorem 3, the existence of a function $\bar{\gamma} \in L^{\infty}(\Omega)$, with $0 \leq \bar{\gamma} \leq 1$ a.e. such that the sequence $\left(\gamma\left(s_{n}\right)\right)_{n \in \mathbb{N}}$ converges to $\bar{\gamma}$ for the weak $\star$ topology of $L^{\infty}(\Omega)$ and 


$$
\begin{aligned}
& \int_{\Omega} \frac{1}{1-\Lambda}\left(M(x)-\Lambda k_{1}(1) I_{N}\right) \nabla \bar{u}(x) \cdot \nabla \bar{v}(x) d x= \\
& \int_{\Omega}\left(\gamma(\bar{s}(x))(\bar{f}(x))^{+}-\bar{\gamma}(x)(\bar{f}(x))^{-}\right) \bar{v}(x) d x, \quad \forall \bar{v} \in H_{0}^{1}(\Omega) .
\end{aligned}
$$

Note that $k_{1}(\cdot)=\lambda(\cdot) \gamma(\cdot)=\frac{1}{1-\Lambda}\left(\lambda(\cdot)-\Lambda k_{1}(1)\right)$.

Some numerical results (given in figures 2.1 and 2.2, on a triangular mesh with 1400 triangles) have been obtained in the following case:

- $N=2$,

- $\Omega=[0,1] \times[0,1]$,

- $\gamma$ and $\lambda$ are obtained from (2.17) with $k_{1}(s)=s$ and $1 / \Lambda=10,100,1000$,

- $\bar{f}(x)=1$ if $x \in[0 ., 0.3] \times[0.7,1],. \bar{f}(x)=0.01$ if $x \in[0.7,1] \times.[0 ., 0.3], \bar{f}(x)=0$ elsewhere,

- $\bar{s}(x)=1$ if $x \in[0 ., 0.3] \times[0.7,1],. \bar{s}(x)=0$ if $x \in[0.7,1] \times.[0 ., 0.3]$,

- since all the resulting discrete pressures are positives, no value is requested for $\tilde{s}$.
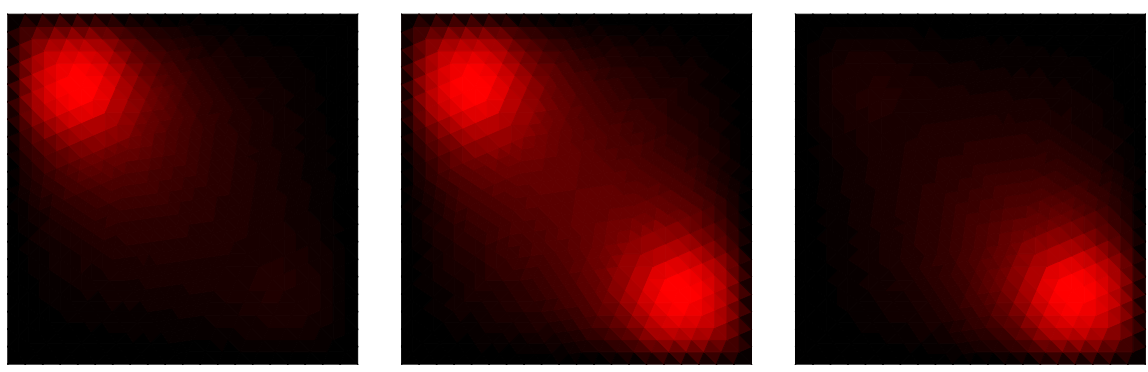

FIG. 2.3. Pressures u for $1 / \Lambda=10,100,1000$.
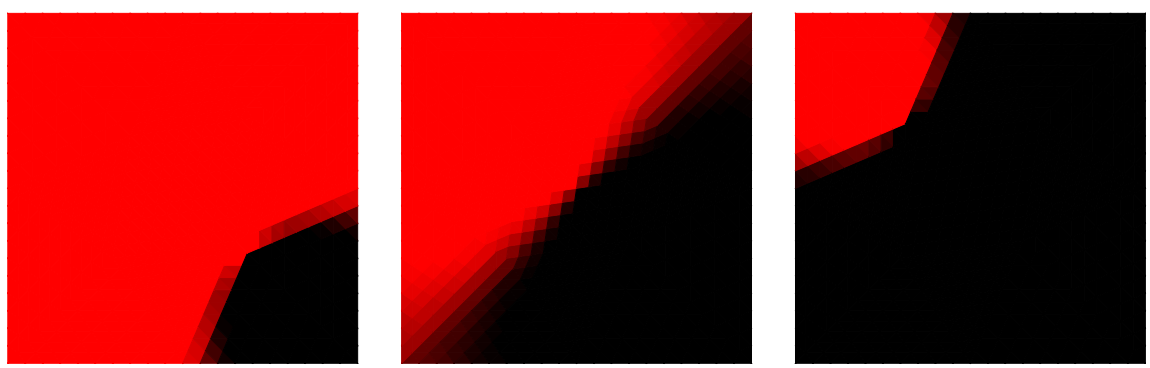

FIG. 2.4. Saturations $s$ for $1 / \Lambda=10,100,1000$.

These results show the difference of behaviours of the two fluids, the mobility of the fluid 2 being largely lower than that of the fluid 1. These result seem to suggest that a stronger result of convergence should be obtained for the saturation (the limit between a region where $s=1$ and $s=0$ seem to be very regular). However, the way to prove this result does not clearly appears.

3. The transient case. We now study the transient case. We first state the continuous result which extends that of Section 2.1. For this purpose, we give some complete formulation of a linear parabolic problem with homogeneous boundary conditions. Let $\Omega$ be an open bounded subset of $\mathbb{R}^{N}$, with $N \in \mathbb{N}_{*}$ and let $\alpha$ and $\beta$ be two real numbers, with $0<\alpha \leq \beta$. We denote by $\mathcal{M}(\alpha, \beta, \Omega, T)$ the set of measurable functions 
$M: \Omega \times(0, T) \longrightarrow \mathcal{L}\left(\mathbb{R}^{N}, \mathbb{R}^{N}\right)$ such that, for a.e. $(x, t) \in \Omega \times(0, T),\|M(x, t)\| \leq \beta$ and for all $(\xi, \chi) \in\left(\mathbb{R}^{N}\right)^{2}, \alpha|\xi|^{2} \leq M(x, t) \xi \cdot \xi$. For a given source term $b \in L^{2}\left(0, T ; H^{-1}(\Omega)\right)$, an initial value $u_{0} \in L^{2}(\Omega)$ and a given $M \in \mathcal{M}(\alpha, \beta, \Omega, T)$, we denote by $\mathcal{F}\left(b, u_{0}, M\right)$ the unique solution $\bar{u}$ of

$$
\begin{aligned}
& \bar{u} \in L^{2}\left(0, T ; H_{0}^{1}(\Omega)\right) \text { and } \bar{u}_{t} \in L^{2}\left(0, T ; H^{-1}(\Omega)\right) \\
& \bar{u}(\cdot, 0)=u_{0} \\
& \left\langle\bar{u}_{t}, \bar{v}\right\rangle+\int_{\Omega \times(0, T)} M(x, t) \nabla \bar{u}(x, t) \cdot \nabla \bar{v}(x, t) d x=\langle b, \bar{v}\rangle, \forall \bar{v} \in L^{2}\left(0, T ; H_{0}^{1}(\Omega)\right) .
\end{aligned}
$$

(recall that a function $\bar{u}$ such that $\bar{u} \in L^{2}\left(0, T ; H_{0}^{1}(\Omega)\right)$ and $\bar{u}_{t} \in L^{2}\left(0, T ; H^{-1}(\Omega)\right)$ can be identified as an element of $\left.C^{0}\left([0, T], L^{2}(\Omega)\right)\right)$. The following result is in particular given in [18] (in which it is called GP-convergence) for more general nonlinear configurations. Note that the framework of [17] only applied to parabolic operators which do not depend on $t$.

THEOREM 5 (Transient H-convergence). Let $\Omega$ be an open bounded subset of $\mathbb{R}^{N}$, with $N \in \mathbb{N}_{*}$ and let $T>0$. Let two real numbers $\alpha$ and $\beta$ be such that $0<\alpha \leq \beta$. Let $\left(M_{n}\right)_{n \in \mathbb{N}}$ be a sequence of elements of $M_{n} \in \mathcal{M}(\alpha, \beta, \Omega, T)$.

Then there exists a subsequence of $\left(M_{n}\right)_{n \in \mathbb{N}}$, again denoted $\left(M_{n}\right)_{n \in \mathbb{N}}$, and a function $M \in \mathcal{M}\left(\alpha, \beta^{2} / \alpha, \Omega, T\right)$ such that:

- for all $b \in L^{2}\left(0, T ; H^{-1}(\Omega)\right)$, and $u_{0} \in L^{2}(\Omega), \mathcal{F}\left(b, u_{0}, M_{n}\right)$ weakly converges to $\mathcal{F}\left(b, u_{0}, M\right)$ in $L^{2}\left(0, T ; H_{0}^{1}(\Omega)\right)$ and $\mathcal{F}\left(b, u_{0}, M_{n}\right)_{t}$ weakly converges to $\mathcal{F}\left(b, u_{0}, M\right)_{t}$ in $L^{2}\left(0, T ; H^{-1}(\Omega)\right)$ as $n \longrightarrow \infty$,

- for all $b \in L^{2}\left(0, T ; H^{-1}(\Omega)\right)$, and $u_{0} \in L^{2}(\Omega), M_{n} \nabla \mathcal{F}\left(b, u_{0}, M_{n}\right)$ weakly converges to $M \nabla \mathcal{F}\left(b, u_{0}, M\right)$ in $\left(L^{2}(\Omega \times(0, T))\right)^{N}$ as $n \longrightarrow \infty$.

We then say that the sequence $\left(M_{n}\right)_{n \in \mathbb{N}} H$-converges to $M$, called the H-limit of the sequence.

REMARK 6. If the functions $M_{n}$ do not depend on $t$, then it can be shown that the obtained H-limit is the same as that which is given by the elliptic problem (this has been proven in [17]).

REMARK 7. There is no evidence, if the $M_{n}(x, t)$ are symmetric, that the H-limit obtained in the transient parabolic case is symmetric.

It is then possible, using finite volume schemes, to develop a discrete framework for the parabolic problem, and then to get the analogous theorems of Hd-convergence (that we do not give in more detail, since it follows similar ideas to that of the steady case). Then we can study the convergence of the finite volume approximation of the solution $(u, s)$ of the transient two-phase flow problem:

$$
\begin{aligned}
& {\left[\left(\Phi_{0}+\kappa u\right) s\right]_{t}-\operatorname{div}[\gamma(s) \lambda(s) \nabla u]=} \\
& {\left[(\bar{u}-u)^{+} \gamma(\bar{s})-(\bar{u}-u)^{-} \gamma(s)\right] \omega} \\
& {\left[\left(\Phi_{0}+\kappa u\right)(1-s)\right]_{t}-\operatorname{div}[(1-\gamma(s)) \lambda(s) \nabla u]=} \\
& {\left[(\bar{u}-u)^{+}(1-\gamma(\bar{s}))-(\bar{u}-u)^{-}(1-\gamma(s))\right] \omega}
\end{aligned}
$$

with the boundary conditions

$$
\begin{aligned}
& u=0 \text { a.e. in } \partial \Omega \times(0, T) \\
& s=\hat{s} \text { a.e. in }\left\{(x, t) \in \partial \Omega \times(0, T), \nabla u(x, t) \cdot \mathbf{n}_{\partial \Omega}(x) \geq 0\right\} .
\end{aligned}
$$

and the initial conditions

$$
\begin{aligned}
& u(\cdot, 0)=u_{0} \text { a.e. in } \Omega, \\
& s(\cdot, 0)=s_{0} \text { a.e. in } \Omega .
\end{aligned}
$$

In (3.1)-(3.3), the following hypotheses are made. 
- the function $\omega \in L^{2}(\Omega \times(0, T))$, which represents a volumic approximation of the well productivity index, is non negative,

- the function $\bar{u} \in L^{\infty}(\Omega \times(0, T))$, which represents the imposed pressure at the wells, is such that there exists two reals $u_{m}$ and $u_{M}$ with $u_{m} \leq \bar{u} \leq u_{M}$ a.e. in $\Omega \times(0, T)$,

- the function $u_{0} \in L^{\infty}(\Omega)$, which is the initial pressure, is such that $u_{m} \leq u_{0} \leq u_{M}$ a.e. in $\Omega$,

- $\Phi_{0}>0$ and $\kappa>0$ are such that $\Phi_{0}-\kappa \max \left(u_{M},-u_{m}\right)>0$,

- $\bar{s} \in L^{\infty}(\Omega \times(0, T))$ with $0 \leq \bar{s} \leq 1$ a.e., and $\hat{s} \in L^{\infty}(\partial \Omega \times(0, T))$ with $0 \leq \hat{s} \leq 1$ a.e.,

- $s_{0} \in L^{\infty}(\Omega)$ with $0 \leq s_{0} \leq 1$ a.e. ( $s_{0}$ is the initial saturation).

Let $\mathcal{D}$ be an admissible discretization of $\Omega$ in the sense given in section 1 . Let $T>0$, let $t^{(0)}=0<t^{(1)}<\ldots t^{(N+1)}=T$. We denote by $\llbracket 0, N \rrbracket$ the set of the integer values $0,1 \ldots N$, we define $\Delta t^{(n)}=t^{(n+1)}-t^{(n)}$ for all $n \in \llbracket 0, N \rrbracket$ and we set

$$
\begin{array}{lll}
\omega_{K}^{(n+1)}=\int_{t^{(n)}}^{t^{(n+1)}} \int_{K} \omega(x, t) d x d t, & & \forall K \in \mathcal{T}, \forall n \in \llbracket 0, N \rrbracket, \\
\bar{u}_{K}^{(n+1)}=\frac{1}{m_{K} \Delta t^{(n)}} \int_{t^{(n)}}^{t^{(n+1)}} \int_{K} \bar{u}(x, t) d x d t, & & \forall K \in \mathcal{T}, \forall n \in \llbracket 0, N \rrbracket, \\
\bar{s}_{K}^{(n+1)}=\frac{1}{m_{K} \Delta t^{(n)}} \int_{t^{(n)}}^{t^{(n+1)}} \int_{K} \bar{s}(x, t) d x d t, & & \forall K \in \mathcal{T}, \forall n \in \llbracket 0, N \rrbracket, \\
\hat{s}_{\sigma}^{(n+1)}=\frac{1}{m_{\sigma} \Delta t^{(n)}} \int_{t^{(n)}}^{t^{(n+1)}} \int_{\sigma} \hat{s}(x, t) d \gamma(x) d t, & & \forall \sigma \in \mathcal{E}_{\mathrm{ext}}, \forall n \in \llbracket 0, N \rrbracket, \\
s_{K}^{(0)}=\frac{1}{\mathrm{~m}_{K}} \int_{K} s_{0}(x) d x, & & \forall K \in \mathcal{T}, \\
u_{K}^{(0)}=\frac{1}{\mathrm{~m}_{K}} \int_{K}^{u_{0}}(x) d x, & & \forall K \in \mathcal{T} .
\end{array}
$$

Then the following numerical scheme is used to build the approximation

$\left(u^{(n+1)}, s^{(n+1)}\right) \in H_{\mathcal{D}}(\Omega) \times L_{\mathcal{D}}(\Omega,[0,1])$, for all $n \in \llbracket 0, N \rrbracket$ : using the upstream weighting scheme defined by the functions $s_{\sigma}(u, s, \hat{s})$ (defined by $(2.13)$ ) and $\mu_{\sigma}(u, s, \hat{s})$ (defined by (2.14)), we denote, for the sake of simplicity,

$$
s_{\sigma}^{(n+1)}=s_{\sigma}\left(u^{(n+1)}, s^{(n+1)}, \hat{s}^{(n+1)}\right) \text { and } \mu_{\sigma}^{(n+1)}=\mu_{\sigma}\left(u^{(n+1)}, s^{(n+1)}, \hat{s}^{(n+1)}\right),
$$

and we set

$$
\begin{aligned}
& \kappa \mathrm{m}_{K}\left(u_{K}^{(n+1)}-u_{K}^{(n)}\right)- \\
& \left.\Delta t^{(n)} \sum_{\sigma \in \mathcal{E}_{K}}^{(n+1)} \tau_{K, \sigma}^{(n+1)}-u_{\sigma}^{(n+1)}\right)=\left(\bar{u}_{K}^{(n+1)}-u_{K}^{(n+1)}\right) \omega_{K}^{(n+1)}, \\
& \forall K \in \mathcal{T}, \forall n \in \llbracket 0, N \rrbracket
\end{aligned}
$$

and

$$
\begin{aligned}
& \mathrm{m}_{K}\left(\Phi_{0}+\kappa u_{K}^{(n+1)}\right) s_{K}^{(n+1)}-\left(\Phi_{0}+\kappa u_{K}^{(n)}\right) s_{K}^{(n)}- \\
& -\Delta t^{(n)} \sum_{\sigma \in \mathcal{E}_{K}} \gamma\left(s_{\sigma}^{(n+1)}\right) \mu_{\sigma}^{(n+1)} \tau_{K, \sigma}\left(u_{\sigma}^{(n+1)}-u_{K}^{(n+1)}\right)= \\
& {\left[\gamma\left(\bar{s}_{K}^{(n+1)}\right)\left(\bar{u}_{K}^{(n+1)}-u_{K}^{(n+1)}\right)^{+}-\gamma\left(s_{K}^{(n+1)}\right)\left(\bar{u}_{K}^{(n+1)}-u_{K}^{(n+1)}\right)^{-}\right] \omega_{K}^{(n+1)},} \\
& \forall K \in \mathcal{T}, \forall n \in \llbracket 0, N \rrbracket .
\end{aligned}
$$

The convergence in $L^{2}(\Omega \times(0, T))$ of the approximate pressure produces the strong convergence in $L^{2}\left(0, T ; H^{-1}(\Omega)\right)$ of the right hand side, which is sufficient to apply the Hdconvergence theorem which mimics Theorem 5 . We then get, similarly to the steady case, a Hd-limit for a subsequence of the approximate pressures. Since a maximum principle applies to the pressure, due to the particular form of the right hand side of (3.1), we get 
that the discrete pressure satisfies $|u| \leq \max \left(u_{M},-u_{m}\right)$. Therefore an $L^{\infty}$ estimate on the approximation of $s$ can be derived (such an estimate is used to prove the existence of a discrete solution) and again a convergence property in the weak $\star$ topology of $L^{\infty}(\Omega \times(0, T))$ is available. However, as in the steady case, no limit equation can be found for the saturation without a strong convergence property.

4. Concluding remarks. The notion of Hd-convergence, developed in this paper, gives a useful tool for studying the convergence of a discrete finite volume scheme, used for the approximation of a two-phase flow in a porous medium. The proof of the Hd-convergence theorem mimics that of the H-convergence theorem; however, although the methods are similar, the limits can be different. This discrete tool is therefore adapted to the case of a coupled discretization: the discrete pressure field is solution of a discrete scheme for an elliptic equation, the coefficients of which result from another discrete scheme in the same grid.

This tool thus helps to get the limit problem of which the limit of the approximate pressure is solution. A weak limit also exists for the saturation since the discrete values are bounded as well as the continuous ones. At this point, we are not able to link the Hd-limit of the sequence of discrete total mobilities and a convenient limit of the sequence of saturations.

\section{REFERENCES}

[1] Alt, H.W., Luckhaus, S., Visintin, A., On nonstationary flow through porous media, Ann. Mat. Pura. Appl., 136 (1984), 303-316.

[2] Alt, H.W., Di Benedetto, E., Nonsteady flow of water and oil through inhomogeneous porous media, Ann. Scuola Norm. Sup. Pisa, 12 (1985), 335-392.

[3] Aziz, K, Settari, A., Petroleum Reservoir Simulation, Applied Science (1979), London.

[4] Carrillo, J., Entropy solutions for nonlinear degenerate problems, Arch. Rat. Mech. Anal., 147 (1999), 269-361.

[5] Chen, Z., Degenerate Two-Phase Incompressible Flow, J. of Differential Equations, 171 (2001), 203-232.

[6] Droniou, J., Gallouët, T., Finite volume methods for convection-diffusion equations with right-hand side in $H^{-1}, M^{2} A N, 36$ (2002), 705-724.

[7] Eymard, R., GallouËt, T., Convergence d'un schéma de type éléments finis-volumes finis pour un système formé d'une équation elliptique et d'une équation hyperbolique, $M^{2} A N$, 27 (1993), 843-861.

[8] Eymard, R., GallouËt, T., H-convergence and numerical schemes for elliptic problems, accepted for publication in SIAM J. of Num. Anal. (2002).

[9] Eymard, R., Gallouët, T., Herbin, R., Finite Volume Methods, Handbook of Numerical Analysis, P.G. Ciarlet and J.L. Lions. eds., VII (2000) 723-1020.

[10] Eymard, R., Gallouët, T., Herbin, R., Michel, A., Convergence of a finite volume scheme for nonlinear degenerate parabolic equations Numer. Math. 92 (2002), 41-82.

[11] Gagneux, G., Madaune-Tort, M., Analyse mathématique de modèles non linéaires de l'ingénierie pétrolière, Mathématiques et Applications 22, Springer-Verlag (1996).

[12] KozLov, S.M., Averaging of difference schemes, Math. USSR Sb 57 (1987), 351-369.

[13] Krushkov, S.N., First order quasilinear equations with several space variables, Math. USSR. Sb. 10 (1970), 217-243.

[14] Murat, F., H-convergence, Séminaire d'analyse fonctionnelle et numérique de l'Université d'Alger (1977-1978).

[15] Ohlberger, M., Convergence of a mixed finite element-finite volume method for the two phase flow in porous media, East-West J. Numer. Math. 5 (1997), 183-210.

[16] Piatnitski, A. RÉmy, E., Homogenization of elliptic difference operators, SIAM J. Math. Anal. 33, 1 (2001), 53-83.

[17] Spagnolo, S., Sulla convergenza di soluzioni di equazioni paraboliche ed ellittiche, Ann. Scuola Norm. Sup. Pisa, Sci. Fis. Mat., III. Ser. 22 (1968), 571-597.

[18] Svanstedt, N., G-convergence of parabolic operators, Nonlinear Anal., Theory Methods Appl. 7 (1999) 807-842. 
[19] Tartar, L., Cours Peccot, Collège de France (Paris, 1977).

[20] Tartar, L., Homogénéisation et H-mesures. (Homogenization and H-measures). (French) ESAIM, Proc. 6 (1999) 111-131.

[21] Vignal, M.H., Convergence of a finite volume scheme for an elliptic-hyperbolic system, $M^{2} A N$, 30, (1996), 841-872. 